MATHEMATICS OF COMPUTATION

Volume 72, Number 243, Pages 1369-1377

S 0025-5718(03)01530-8

Article electronically published on March 26, 2003

\title{
PIECEWISE QUADRATIC TRIGONOMETRIC POLYNOMIAL CURVES
}

\author{
XULI HAN
}

\begin{abstract}
Analogous to the quadratic B-spline curve, a piecewise quadratic trigonometric polynomial curve is presented in this paper. The quadratic trigonometric polynomial curve has $C^{2}$ continuity, while the quadratic B-spline curve has $C^{1}$ continuity. The quadratic trigonometric polynomial curve is closer to the given control polygon than the quadratic B-spline curve.
\end{abstract}

\section{INTRODUCTION}

It is well-known that polynomial B-splines, particular the quadratic and cubic Bsplines, have gained widespread application. The purpose of this paper is to present a practical quadratic trigonometric polynomial curve analogous to the quadratic Bspline curve.

The trigonometric B-splines were introduced in 8], and the recurrence relation for the trigonometric B-splines of arbitrary order was established in [5]. It was further shown in [10] that the trigonometric B-splines of odd order form a partition of a constant in the case of equidistant knots, and therefore the corresponding trigonometric B-spline curve possesses the convex hull property. There are other papers in which the trigonometric B-splines and polynomials have been studied, see references therein. In this paper, a different quadratic trigonometric polynomial curve is presented. The curve has $C^{2}$ continuity with a nonuniform knot vector.

This paper is organized as follows. In section 2 , the basis functions of the quadratic trigonometric polynomial curves are described and the properties of the basis functions are shown. In section 3, the quadratic trigonometric polynomial curve is given. It is shown in section 4 that the quadratic trigonometric polynomial curve is closer to the given control polygon than the quadratic B-spline curve.

\section{QUADRATIC TRIGONOMETRIC BASIS FUNCTIONS}

\subsection{Construction of the basis functions.}

Definition 1. Given knots $u_{0}<u_{1}<\cdots<u_{n+3}$, let $\Delta u_{i}=u_{i+1}-u_{i}$,

$$
\alpha_{i}=\frac{1}{3} \frac{\Delta u_{i}}{\Delta u_{i-1}+\Delta u_{i}}, \quad \beta_{i}=\frac{1}{3} \frac{\Delta u_{i}}{\Delta u_{i}+\Delta u_{i+1}}, \quad t_{i}(u)=\frac{\pi}{2} \frac{u-u_{i}}{\Delta u_{i}},
$$

Received by the editor November 30, 2000 and, in revised form, November 7, 2001.

2000 Mathematics Subject Classification. Primary 65D17, 65D10; Secondary 42A10.

Key words and phrases. Trigonometric polynomial, trigonometric curve, splines.

This work was conducted while the author was visiting the geometric modeling group at the University of Florida. 


$$
\begin{aligned}
c(t) & =(1-\sin t)(1-\sin t+2 \cos t), \\
d(t) & =(1-\cos t)(1-\cos t+2 \sin t) .
\end{aligned}
$$

Then the associated normalized quadratic trigonometric basis functions are defined to be the following functions:

$$
b_{i}(u)= \begin{cases}\beta_{i} d\left(t_{i}\right), & u \in\left[u_{i}, u_{i+1}\right), \\ 1-\alpha_{i+1} c\left(t_{i+1}\right)-\beta_{i+1} d\left(t_{i+1}\right), & u \in\left[u_{i+1}, u_{i+2}\right), \\ \alpha_{i+2} c\left(t_{i+2}\right), & u \in\left[u_{i+2}, u_{i+3}\right), \\ 0, & u \notin\left[u_{i}, u_{i+3}\right),\end{cases}
$$

for $i=0,1, \ldots, n$.

Theorem 1. The basis functions have the following properties:

a) $b_{i}(u)>0$, for $u_{i}<u<u_{i+3}$,

b) $b_{i}(u)=0$, for $u_{0} \leq u \leq u_{i}, u_{i+3} \leq u \leq u_{n+3}$,

c) $\sum_{i=0}^{n} b_{i}(u)=1, u \in\left[u_{2}, u_{n+1}\right]$.

Proof. Let $\gamma_{i+1}=\max \left\{\alpha_{i+1}, \beta_{i+1}\right\}$. Then

$$
\begin{aligned}
\alpha_{i+1} c\left(t_{i+1}\right)+\beta_{i+1} d\left(t_{i+1}\right) & \leq \gamma_{i+1}\left(c\left(t_{i+1}\right)+d\left(t_{i+1}\right)\right)=\gamma_{i+1}\left(3-2 \sin 2 t_{i+1}\right) \\
& \leq 3 \gamma_{i+1}<1 .
\end{aligned}
$$

Thus $b_{i}(u)>0$, for $u \in\left[u_{i+1}, u_{i+2}\right)$.

For $u \in\left[u_{i}, u_{i+1}\right), i=2,3, \ldots, n$, let

$$
\begin{aligned}
& b_{i 0}\left(t_{i}\right)=\alpha_{i} c\left(t_{i}\right), \\
& b_{i 1}\left(t_{i}\right)=1-\alpha_{i} c\left(t_{i}\right)-\beta_{i} d\left(t_{i}\right), \\
& b_{i 2}\left(t_{i}\right)=\beta_{i} d\left(t_{i}\right) .
\end{aligned}
$$

Then $b_{i-2}(u)=b_{i 0}\left(t_{i}\right), b_{i-1}(u)=b_{i 1}\left(t_{i}\right), b_{i}(u)=b_{i 2}\left(t_{i}\right), b_{j}(u)=0(j \neq i-2$, $i-1, i)$, and

$$
\sum_{j=0}^{n} b_{j}(u)=b_{i 0}\left(t_{i}\right)+b_{i 1}\left(t_{i}\right)+b_{i 2}\left(t_{i}\right)=1 .
$$

The remaining cases follow obviously.

Remark. In view of the properties a)-c), we say that the basis functions form a partition of unity and the function $b_{i}(u)$ has a support on the interval $\left[u_{i}, u_{i+3}\right]$.

For equidistant knots, we refer to the $b_{i}(u)$ as a uniform basis function. Figure 1 shows the graph of three uniform basis functions. The basis functions defined over nonequidistant knots are called nonuniform basis functions.

2.2. Continuity of the basis functions. It is known that the quadratic B-splines have $C^{1}$ continuity. The following theorem shows the continuity of the quadratic trigonometric basis functions.

Theorem 2. The quadratic trigonometric basis function $b_{i}(u)$ has $C^{2}$ continuity at each of the knots. 


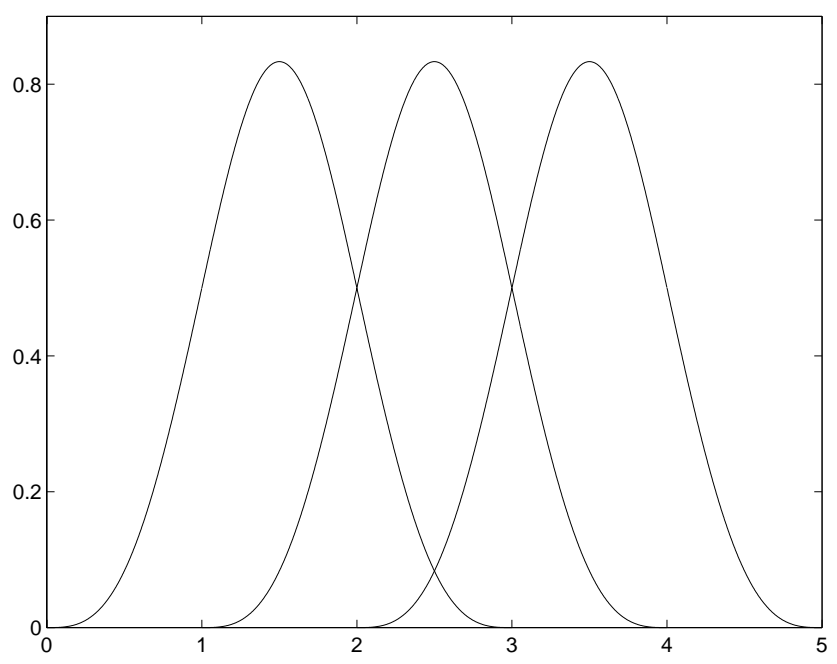

FiguRE 1. Uniform basis functions.

Proof. Consider the continuity at the knots $u_{i+1}$ and $u_{i+2}$ (We can deal with the knots $u_{i}$ and $u_{i+3}$ in the same way). Since

$$
\begin{aligned}
& c(t)=\frac{3}{2}-2 \sin t+2 \cos t-\sin 2 t-\frac{1}{2} \cos 2 t, \\
& d(t)=\frac{3}{2}+2 \sin t-2 \cos t-\sin 2 t+\frac{1}{2} \cos 2 t,
\end{aligned}
$$

straightforward computation gives

$$
\begin{array}{ll}
b_{i}\left(u_{i+1}^{-}\right)=3 \beta_{i}, & b_{i}\left(u_{i+1}^{+}\right)=1-3 \alpha_{i+1}, \\
b_{i}\left(u_{i+2}^{-}\right)=1-3 \beta_{i+1}, & b_{i}\left(u_{i+2}^{+}\right)=3 \alpha_{i+2}, \\
b_{i}^{\prime}\left(u_{i+1}^{-}\right)=\frac{2 \pi \beta_{i}}{\Delta u_{i}}, & b_{i}^{\prime}\left(u_{i+1}^{+}\right)=\frac{2 \pi \alpha_{i+1}}{\Delta u_{i+1}}, \\
b_{i}^{\prime}\left(u_{i+2}^{-}\right)=-\frac{2 \pi \beta_{i+1}}{\Delta u_{i+1}}, & b_{i}^{\prime}\left(u_{i+2}^{+}\right)=-\frac{2 \pi \alpha_{i+2}}{\Delta u_{i+2}}, \\
b_{i}^{\prime \prime}\left(u_{i+1}^{-}\right)=b_{i}^{\prime \prime}\left(u_{i+1}^{+}\right)=0, \\
b_{i}^{\prime \prime}\left(u_{i+2}^{-}\right)=b_{i}^{\prime \prime}\left(u_{i+2}^{+}\right)=0 .
\end{array}
$$

Since

$$
\begin{gathered}
3 \alpha_{j+1}=1-3 \beta_{j}=\frac{\Delta u_{j+1}}{\Delta u_{j}+\Delta u_{j+1}}, \\
\frac{\alpha_{j+1}}{\Delta u_{j+1}}=\frac{\beta_{j}}{\Delta u_{j}}=\frac{1}{3} \frac{1}{\Delta u_{j}+\Delta u_{j+1}},
\end{gathered}
$$

we have

$$
b_{i}^{(k)}\left(u_{i+1}^{-}\right)=b_{i}^{(k)}\left(u_{i+1}^{+}\right), \quad b_{i}^{(k)}\left(u_{i+2}^{-}\right)=b_{i}^{(k)}\left(u_{i+2}^{+}\right), \quad k=0,1,2 .
$$

The theorem follows. 


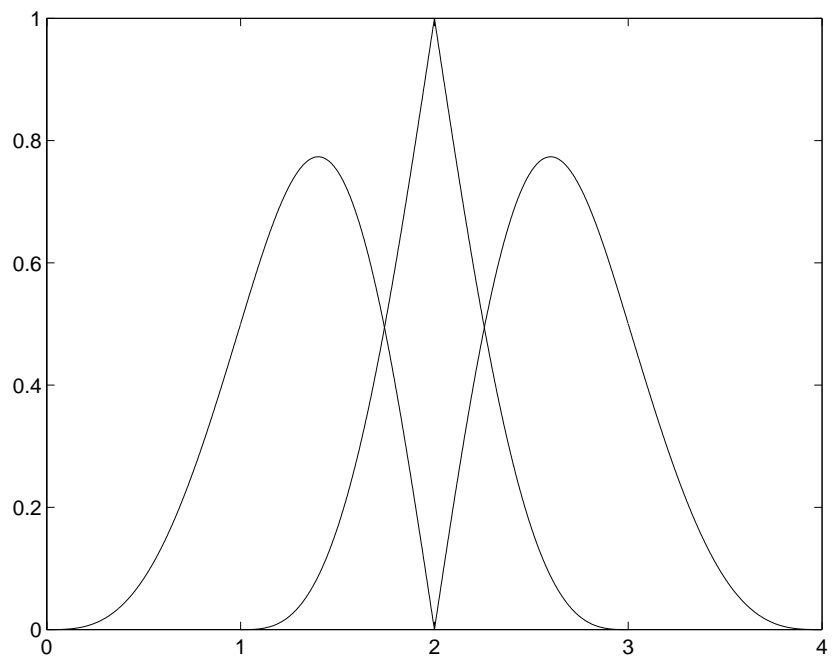

FiguRE 2. The basis functions with a double knot.

2.3. The case of multiple knots. So far in the discussion of the basis functions, we have assumed that each point is simple. On the other hand, the basis functions also make sense when knots are considered with multiplicity $k \leq 3$. For trigonometric basis functions with multiple knots, it is worth noting that we shrink the corresponding intervals to zero and drop the corresponding pieces. For example, if $u_{i+1}=u_{i+2}$ is a double knot, then we define

$$
b_{i}(u)= \begin{cases}\beta_{i} d\left(t_{i}\right), & u \in\left[u_{i}, u_{i+1}\right), \\ \alpha_{i+2} c\left(t_{i+2}\right), & u \in\left[u_{i+2}, u_{i+3}\right), \\ 0, & u \notin\left[u_{i}, u_{i+3}\right) .\end{cases}
$$

Theorem 3. Suppose that a basis function has a knot of multiplicity $k=2$ or 3 at a parameter value $u$. Then at this point the continuity of the basis function is reduced from $C^{2}$ to $C^{2-k}\left(C^{-1}\right.$ means discontinuous $)$. Moreover, the support interval of the basis function is reduced from 3 segments to $4-k$ segments.

Proof. This is a direct application of (1) and the expressions given in the proof of Theorem 2.

Theorem 3 deals with the geometric significance of multiple knots. For applications, it is important to use multiple knots. Figure 2 shows the trigonometric basis functions with a double knot.

\section{QuAdratic TRIGONOMETRIC POLYNOMIAL CURVES}

Definition 2. Given points $P_{i}(i=0,1, \ldots, n)$ in $\mathbb{R}^{2}$ or $\mathbb{R}^{3}$ and a knot vector $U=\left(u_{0}, u_{1}, \ldots, u_{n+3}\right)$, we call

$$
T(u)=\sum_{j=0}^{n} b_{j}(u) P_{j}, \quad n \geq 2, u \in\left[u_{2}, u_{n+1}\right],
$$

a quadratic trigonometric polynomial curve. 
Remark. If $u_{i} \neq u_{i+1}(2 \leq i \leq n)$, then for $u \in\left[u_{i}, u_{i+1}\right]$, the curve $T(u)$ can be represented by the curve segment

$$
T_{i}\left(t_{i}\right)=b_{i 0}\left(t_{i}\right) P_{i-2}+b_{i 1}\left(t_{i}\right) P_{i-1}+b_{i 2}\left(t_{i}\right) P_{i},
$$

where $b_{i 0}\left(t_{i}\right), b_{i 1}\left(t_{i}\right), b_{i 2}\left(t_{i}\right)$ are given by $(2),(3),(4)$, respectively.

Analogously to the quadratic B-spline curve, the choice of the knot vector automatically determines the continuity of the quadratic trigonometric polynomial curve at each of the knots, as shown by the following theorem.

Theorem 4. If a knot $u_{i}$ has multiplicity $k=1,2$, or 3 , then the quadratic trigonometric polynomial curve has $C^{2}$ continuity for $k=1$ and $C^{2-k}$ continuity for $k=2$ or 3 at $u_{i}$. Moreover,

$$
\begin{aligned}
T\left(u_{i}^{-}\right) & =\left(1-3 \beta_{i-1}\right) P_{i-2}+3 \beta_{i-1} P_{i-1}, \\
T\left(u_{i}^{+}\right) & =3 \alpha_{i} P_{i-2}+\left(1-3 \alpha_{i}\right) P_{i-1}, \\
T^{\prime}\left(u_{i}^{-}\right) & =\frac{2 \pi \beta_{i-1}}{\Delta u_{i-1}}\left(P_{i-1}-P_{i-2}\right), \\
T^{\prime}\left(u_{i}^{+}\right) & =\frac{2 \pi \alpha_{i}}{\Delta u_{i}}\left(P_{i-1}-P_{i-2}\right), \\
T^{\prime \prime}\left(u_{i}^{-}\right) & =T^{\prime \prime}\left(u_{i}^{+}\right)=0 .
\end{aligned}
$$

Proof. This is a direct consequence of Theorems 2 and 3.

Remark. Since $T^{\prime \prime}\left(u_{i}^{-}\right)=T^{\prime \prime}\left(u_{i}^{+}\right)=0$, we can deduce that the curvatures of the quadratic trigonometric polynomial curve at the interior knots are zero.

Remark. The choice of the first and last two knots is free, and these knots can be adjusted to give the desired boundary behavior of the curve. See the following descriptions.

For an open trigonometric polynomial curve, we choose the knot vector

$$
U=\left(u_{0}=u_{1}=u_{2}, u_{3}, \ldots, u_{n}, u_{n+1}=u_{n+2}=u_{n+3}\right) .
$$

This assures that $P_{0}$ and $P_{n}$ are points on the curve. Of course, the interior knots can be multiple knots. In addition, since

$$
\begin{aligned}
T^{\prime}\left(u_{2}^{+}\right) & =\frac{2 \pi}{3 \Delta u_{2}}\left(P_{1}-P_{0}\right), \\
T^{\prime}\left(u_{n+1}^{-}\right) & =\frac{2 \pi}{3 \Delta u_{n}}\left(P_{n}-P_{n-1}\right),
\end{aligned}
$$

the edges $P_{0} P_{1}$ and $P_{n-1} P_{n}$ of the control polygon are tangent to the curve. (If one or more neighboring knots all fall at $P_{0}$, then the tangent at $P_{0}$ corresponds to the edge of the control polygon passing through $P_{0}$ and the first distinct neighbor of $P_{0}$.).

Figure 3 shows an open quadratic trigonometric curve (solid lines) and a quadratic B-spline curve (dashed lines) corresponding to the same control polygon for a nonuniform knot vector $U=(0,0,0,0.5,1.5,2,3,4,4,4)$. 


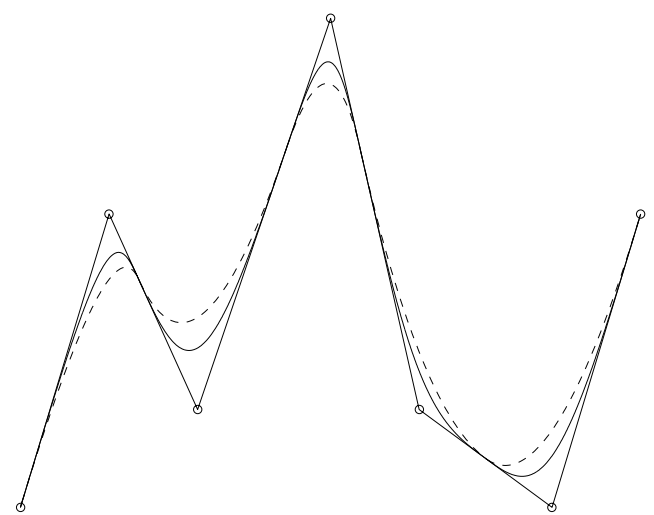

FiguRE 3. Open curves with a nonuniform knot vector.

In order to construct a closed trigonometric curve, we can extend the given points $P_{0}, P_{1}, \ldots, P_{n}$ by setting $P_{n+1}=P_{0}, P_{n+2}=P_{1}$ and expand the knot vector by setting $u_{n+4}=u_{n+3}+\Delta u_{n+2} \Delta u_{2} / \Delta u_{1}$ (so that $\left.T\left(u_{2}\right)=T\left(u_{n+3}\right)\right)$ and $u_{n+5} \leq$ $u_{n+4}$. Thus the parametric formula for a closed quadratic trigonometric curve is

$$
T(u)=\sum_{j=0}^{n+2} b_{j}(u) P_{j}, \quad \text { with } u \in\left[u_{2}, u_{n+3}\right],
$$

where $b_{n+1}$ and $b_{n+2}$ are given by expanding (1).

The parametric formula for a closed quadratic trigonometric curve also can be given by

$$
T(u)=\sum_{j=0}^{n} b_{j}(u) P_{j}, \quad \text { with } u \in\left[u_{0}, u_{n+1}\right]
$$

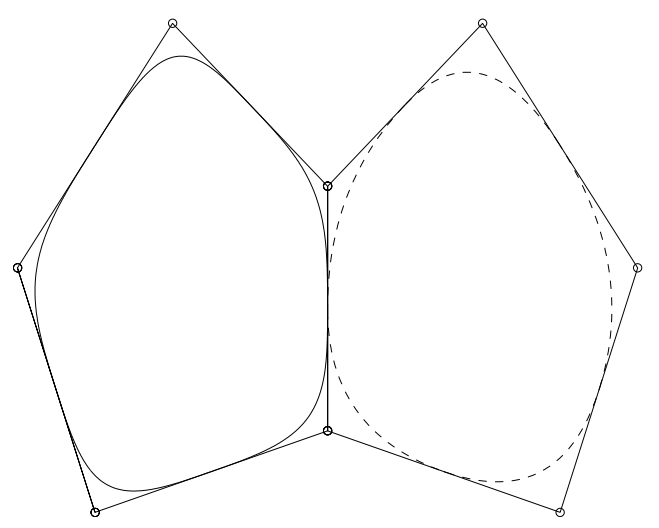

Figure 4. Closed curves with a uniform knot vector. 
here the changes from Definition 1 are to let $\alpha_{0}=\alpha_{n+1}\left(\right.$ so that $\left.T\left(u_{0}\right)=T\left(u_{n+1}\right)\right)$ and

$$
\begin{array}{r}
b_{n-1}(u)= \begin{cases}\beta_{n-1} d\left(t_{n-1}\right), & u \in\left[u_{n-1}, u_{n}\right), \\
1-\alpha_{n} c\left(t_{n}\right)-\beta_{n} d\left(t_{n}\right), & u \in\left[u_{n}, u_{n+1}\right], \\
\alpha_{0} c\left(t_{0}\right), & u \in\left[u_{0}, u_{1}\right), \\
0, & \text { otherwise, }\end{cases} \\
b_{n}(u)= \begin{cases}\beta_{n} d\left(t_{n}\right), & u \in\left[u_{n}, u_{n+1}\right], \\
1-\alpha_{0} c\left(t_{0}\right)-\beta_{0} d\left(t_{0}\right), & u \in\left[u_{0}, u_{1}\right), \\
\alpha_{1} c\left(t_{1}\right), & u \in\left[u_{1}, u_{2}\right), \\
0, & \text { otherwise. }\end{cases}
\end{array}
$$

Figure 4 shows a closed quadratic trigonometric curve (solid lines) and a quadratic B-spline curve (dashed lines) corresponding to the same control polygon for a uniform knot vector.

\section{Approximability}

Control polygons provide an important tool in geometric modeling. It is an advantage if the curve being modeled tends to preserve the shape of its control polygon.

From (6), we have

$$
T_{i}\left(t_{i}\right)=P_{i-1}+b_{i 0}\left(t_{i}\right)\left(P_{i-2}-P_{i-1}\right)+b_{i 2}\left(t_{i}\right)\left(P_{i}-P_{i-1}\right) .
$$

Therefore, the point $T_{i}\left(t_{i}\right)$ (for fixed $u \in\left(u_{i}, u_{i+1}\right)$ ) converges to the point $P_{i-1}$ as $\Delta u_{i} \rightarrow 0$ for fixed $\Delta u_{i-1}$ and $\Delta u_{i+1}$. The curve segment $T_{i}\left(t_{i}\right)\left(u \in\left[u_{i}, u_{i+1}\right]\right)$ tends to be merged with the line segments $T_{i}(0) P_{i-1}$ and $P_{i-1} T_{i}(\pi / 2)$.

From Figures 3 and 4, we can see that the quadratic trigonometric curve is closer to the given control polygon than the quadratic B-spline curve. This fact is proved as follows.

Lemma. For $t \in[0, \pi / 2]$, let

$$
f(t)=c(t)+d(t)+2 \sqrt{c(t) d(t)}
$$

Then $2 \leq f(t) \leq 3, f(t)=2$ if and only if $t=\pi / 4$, and $f(t)=3$ if and only if $t=0$ or $\pi / 2$.

Proof. Let $x=\sin t+\cos t-1$; then $x \in[0, \sqrt{2}-1], c(t)+d(t)=3-2 \sin 2 t=$ $3-2 x(x+2),(1-\sin t)(1-\cos t)=x^{2} / 2,(1-\sin t+2 \cos t)(1-\cos t+2 \sin t)=$ $\left(5 x^{2}+12 x\right) / 2$ and

$$
f(t)=3-x\left(2 x+4-\sqrt{5 x^{2}+12 x}\right) .
$$

While $x \in[0, \sqrt{2}-1]$, we have $\sqrt{5 x^{2}+12 x}<2 x+4$, and then $f(t) \leq 3 ; f(t)=3$ if and only if $x=0$.

On the other hand, while $1-4 x-2 x^{2} \geq 0$, i.e., $x \in[0, \sqrt{6} / 2-1]$, we have $f(t)-2=1-4 x-2 x^{2}+x \sqrt{5 x^{2}+12 x}>0$. While $x \in(\sqrt{6} / 2-1, \sqrt{2}-1]$, since

$$
x^{4}-4 x^{3}-12 x^{2}+8 x-1=\left(x^{2}+2 x-1\right)\left(x^{2}-6 x+1\right) \geq 0,
$$

we have $x \sqrt{5 x^{2}+12 x} \geq 2 x^{2}+4 x-1$, and then $f(t) \geq 2 ; f(t)=2$ if and only if $x=\sqrt{2}-1$. 
Let there be given data points $P_{i} \in \mathbb{R}^{2}$ or $\mathbb{R}^{3}(i=0,1, \ldots, n)$ and knots $u_{0}<$ $u_{1}<\cdots<u_{n+3}$. For $u \in\left[u_{k}, u_{k+1}\right]$, the associated quadratic B-spline curve can be given by

$$
B_{k}(s)=a_{k 0}(s) P_{k-2}+a_{k 1}(s) P_{k-1}+a_{k 2}(s) P_{k},
$$

where $s=\left(u-u_{k}\right) / \Delta u_{k}$ and

$$
\begin{aligned}
& a_{k 0}(s)=3 \alpha_{k}(1-s)^{2}, \\
& a_{k 1}(s)=1-3 \alpha_{k}(1-s)^{2}-3 \beta_{k} s^{2}, \\
& a_{k 2}(s)=3 \beta_{k} s^{2} .
\end{aligned}
$$

Theorem 5. Suppose $P_{k-2}, P_{k-1}, P_{k}$ are not collinear. For given $0 \leq \lambda \leq 1$, let $Q_{k-1}=\lambda P_{k-2}+(1-\lambda) P_{k}, B_{k}(\hat{s})$ and $T_{k}(\hat{t})$ be the intersection points of line segment $P_{k-1} Q_{k-1}$ with the curves $B_{k}(s)$ and $T_{k}(t)$ respectively for $u \in\left[u_{k}, u_{k+1}\right]$. Then

$$
\frac{2}{3}\left\|B_{k}(\hat{s})-P_{k-1}\right\| \leq\left\|T_{k}(\hat{t})-P_{k-1}\right\| \leq\left\|B_{k}(\hat{s})-P_{k-1}\right\| .
$$

Moreover, $2\left\|B_{k}(\hat{s})-P_{k-1}\right\|=3\left\|T_{k}(\hat{t})-P_{k-1}\right\|$ if and only if $u=\left(u_{k}+u_{k+1}\right) / 2$, while $\left\|B_{k}(\hat{s})-P_{k-1}\right\|=\left\|T_{k}(\hat{t})-P_{k-1}\right\|$ if and only if $u=u_{k}$ or $u=u_{k+1}$.

Proof. We can write (9) and (6) as

$$
\begin{gathered}
B_{k}(s)=P_{k-1}+\left(a_{k 0}(s)+a_{k 2}(s)\right)\left(\frac{a_{k 0}(s) P_{k-2}+a_{k 2}(s) P_{k}}{a_{k 0}(s)+a_{k 2}(s)}-P_{k-1}\right), \\
T_{k}(t)=P_{k-1}+\left(b_{k 0}(t)+b_{k 2}(t)\right)\left(\frac{b_{k 0}(t) P_{k-2}+b_{k 2}(t) P_{k}}{b_{k 0}(t)+b_{k 2}(t)}-P_{k-1}\right),
\end{gathered}
$$

where

$$
\left(a_{k 0}(s) P_{k-2}+a_{k 2}(s) P_{k}\right) /\left(a_{k 0}(s)+a_{k 2}(s)\right)
$$

and

$$
\left(b_{k 0}(t) P_{k-2}+b_{k 2}(t) P_{k}\right) /\left(b_{k 0}(t)+b_{k 2}(t)\right)
$$

are points on the line segment $P_{k-2} P_{k}$.

Since $a_{k 0}(s) /\left(a_{k 0}(s)+a_{k 2}(s)\right)$ and $b_{k 0}(t) /\left(b_{k 0}(t)+b_{k 2}(t)\right)$ are monotone for $s \in$ $[0,1]$ and $t \in[0, \pi / 2]$ respectively, there exist unique $\hat{s}$ and $\hat{t}$ such that

$$
\frac{a_{k 0}(\hat{s})}{a_{k 0}(\hat{s})+a_{k 2}(\hat{s})}=\frac{b_{k 0}(\hat{t})}{b_{k 0}(\hat{t})+b_{k 2}(\hat{t})}=\lambda
$$

Thus, we get $a_{k 0}(\hat{s}) b_{k 2}(\hat{t})=a_{k 2}(\hat{s}) b_{k 0}(\hat{t})$, and then $\hat{s}^{2} c(\hat{t})=(1-\hat{s})^{2} d(\hat{t})$, and also

$$
\begin{gathered}
c(\hat{t})=(1-\hat{s})^{2} f(\hat{t}), \quad d(\hat{t})=\hat{s}^{2} f(\hat{t}), \\
b_{k 0}(\hat{t})+b_{k 2}(\hat{t})=\frac{1}{3}\left(a_{k 0}(\hat{s})+b_{k 2}(\hat{s})\right) f(\hat{t}) .
\end{gathered}
$$

Therefore

$$
T_{k}(\hat{t})-P_{k-1}=\frac{1}{3} f(\hat{t})\left(B_{k}(\hat{s})-P_{k-1}\right) .
$$

By the lemma, we have

$$
\frac{2}{3}\left\|B_{k}(\hat{s})-P_{k-1}\right\| \leq\left\|T_{k}(\hat{t})-P_{k-1}\right\| \leq\left\|B_{k}(\hat{s})-P_{k-1}\right\|,
$$

$2\left(\left\|T_{k}(\hat{t})-P_{k-1}\right\|\right) / 3=\left\|B_{k}(\hat{s})-P_{k-1}\right\|$ if and only if $\hat{s}=1 / 2(\hat{t}=\pi / 4)$, while $\left\|T_{k}(\hat{t})-P_{k-1}\right\|=\left\|B_{k}(\hat{s})-P_{k-1}\right\|$ if and only if $\hat{s}=0,1(\hat{t}=0, \pi / 2)$. 


\section{REFERENCES}

[1] J. Hoschek and D. Lasser, Fundamentals of computer aided geometric design (Translated by L. L. Schumaker), A. K. Peters, Wellesley, MA, 1993. MR 94i:65003

[2] P. E. Koch, Multivariate trigonometric B-splines, J. Approx. Theory 54(1988), 162-168. MR 90d: 42004

[3] P. E. Koch, T. Lyche, M. Neamtu, and L. L. Schumaker, Control curves and knot insertion for trigonometric splines, Adv. Comp. Math. 3(1995), 405-424. MR 96k:41012

[4] T. Lyche, A Newton form for trigonometric Hermite interpolation, BIT 19(1979), 229-235. MR 80d:41004

[5] T. Lyche and R. Winther, A stable recurrence relation for trigonometric B-splines, J. Approx. Theory 25(1979), 266-279. MR 81a:42007

[6] T. Lyche and L. L. Schumaker, Quasi-interpolants based on trigonometric splines, J. Approx. Theory 95(1998), 280-309. MR 99i:41001

[7] J. M. Peña, Shape preserving representations for trigonometric polynomial curves, Computer Aided Geometric Design 14(1997), 5-11. MR 97k:65049

[8] I. J. Schoenberg, On trigonometric spline interpolation, J. Math. Mech. 13(1964), 795-825. MR 29:2589

[9] J. Sánchez-Reyes, Harmonic rational Bézier curves, p-Bézier curves and trigonometric polynomials, Computer Aided Geometric Design 15(1998), 909-923. MR 99h:65033

[10] G. Walz, Some identities for trigonometric B-splines with application to curve design, BIT 37(1997), 189-201. MR 97i:41018

[11] G. Walz, Trigonometric Bézier and Stancu polynomials over intervals and triangles, Computer Aided Geometric Design, 14(1997), 393-397. MR 97m:65040

Department of Applied Mathematics and Applied Software, Central South UniverSity, Changsha, 410083, Peoples Republic of China

E-mail address: xlhan@mail.csu.edu.cn 